\title{
PANDEMIA Y RESILIENCIA. APORTACIONES ACADÉMICAS EN TIEMPOS DE CRISIS
}

\author{
PANDEMIC AND RESILIENCE. \\ CONTRIBUTIONS IN TIMES OF CRISIS
}

\author{
Marcin Kazmierczak, María Teresa Signes y Cintia Carreira Zafra (eds.). \\ Pamplona: Eunsa, 2020, 422 págs.
}

Audacter et opportune. Es alentador que haya quien, ante la pandemia, no se limite a lamentarse e incluso se atreva a pensar... Sí, pensar, ese ejercicio cada vez menos frecuente, sustituido en nuestra postmodernidad por los de imaginar y sentir. Como es lógico, las primeras disciplinas científicas en responder al reto de este jinete del Apocalipsis son las agrupadas bajo el marbete «ciencias de la salud». Por eso resulta tan sorprendente e interesante que, desde el ámbito de las humanidades y las ciencias sociales, alguien aporte reflexiones de carácter científico, tras pocos meses del comienzo de esta pesadilla, tal como lo ha hecho el grupo de investigación «Familia, Educación y Escuela Inclusiva» (TRIVIUM), de la Universitat Abat Oliba CEU, de Barcelona.

El estudio de la resiliencia -que algunos insistimos en llamar con su nombre originario y clásico, fortaleza - corresponde en principio a la filosofía práctica; más en concreto, a la ética. No obstante, también desde la psicología positiva se estudia, atendiendo a las capacidades y los principios de los seres humanos, tanto desde una perspectiva individual como colectiva. La resiliencia se orienta al fortalecimiento de la persona y de la comunidad.

Este volumen se estructura en seis bloques temáticos, que corresponden a otras tantas disciplinas: filosofía, literatura, lingüística, ciencias de la educación, psicología y derecho. Los autores de los diecinueve artículos (más un prólogo y un epílogo dedicado a la memoria de César Casimiro, filósofo y educador, fallecido el 20 de mayo de 2020), han sumado esfuerzos para llegar a una interpretación sobre la realidad de la pandemia, sus consecuencias y el desafío que tiene ante sí la comunidad educativa. Uno de los objetivos de esta obra, amén de estimular la reflexión, es fomentar la resiliencia desde todos los niveles de la comunidad educativa.

Aun a riesgo de ser prolijo, procedo a enumerar y valorar brevemente los diecinueve artículos que constituyen el corpus de esta aportación académica.

Los primeros cuatro capítulos reflexionan a partir de la filosofía, ofreciendo una mirada desde el dolor, la esperanza, la educación, la belleza y la virtud. En primer lugar, en «Las competencias clave y los tiempos de crisis, ¿es necesaria una competencia existencial?», Juan Manuel Monfort afirma que la pandemia nos ha hecho más conscientes que nunca de nuestra 
vulnerabilidad, enfrentándonos a nuestra trascendencia y preguntándonos sobre el sentido de la vida y de la muerte. El libro empieza bien, con pensamiento fuerte, contrastando con el pensamiento que predomina en los ámbitos académicos, tan orillado a veces por el débil o líquido.

En la segunda propuesta, «A la búsqueda del ideal», María Victoria Moreno parte de una interesante reflexión sobre qué cosa es la juventud y cómo ayudar a los alumnos a hacer frente a esta situación anómala, creciendo en magnanimidad, vida interior y pasión por la verdad y el bien. La suya es una propuesta desde un humanismo integrador de todas las dimensiones de la persona.

En la misma línea, Jaime Vilarroig nos regala con el cuarto capítulo, titulado «Leibniz, Voltaire y vuelta a Job». Una propuesta audaz, pues tras resumir la hipótesis del mundo mejor de los posibles de Leibniz y el deísmo de Voltaire, propone como modelo resiliente la figura bíblica de Job. Valiente es su consideración del pecado original, verdad vitanda desde el advenimiento de la Modernidad. El del pecado original es el dogma más difícil de entender (tras el de la Santísima Trinidad, claro), pero sin el cual no hay manera de explicar la historia de la humanidad y la condición moral del individuo, que se debate, quiéralo o no, entre el bien y el mal. La Ilustración apostó por el hombre naturalmente bueno y el marxismo cultural por el hombre intrínsecamente malo, pero ni la una ni el otro han ofrecido una explicación mínimamente satisfactoria de esta perpetua contienda.

Completa este primer bloque temático el capítulo tercero, «El poder de la belleza en tiempos de pandemia», de María Teresa Signes, que pone de manifiesto cómo las artes plásticas, la literatura y la música han recuperado unos espacios y un protagonismo inesperado en los días de confinamiento, favoreciendo la resiliencia. Y, tras reflexionar sobre la dimensión estética del hombre, concluye que la educación en la belleza debería tener una especial consideración en el currículo educativo, por lo mucho que aporta al crecimiento personal y a la apertura del espíritu.

Los siguientes capítulos ofrecen reflexiones desde la literatura; los cuatro atienden a la dimensión catártica de la literatura, así como el poder educativo de los distintos personajes de las grandes obras literarias. Suponen la dimensión mimética de la literatura y el valor de la experiencia vicaria: vivimos otras vidas... o, en expresión célebre y feliz, leemos para saber que no estamos solos.

Bajo el título «Alquimistas del dolor. Relatos biográficos en la didáctica de la psicología de la personalidad», Mar Álvarez y Laura Giordano presentan una experiencia de innovación docente en la que convergen dos disciplinas, la psicología y la historia. Desde esa intersección presentan relatos históricos y literarios cuyo hilo conductor, y al mismo tiempo unificador, es la resiliencia.

En segundo lugar, Mariano Bártoli, en «Resistir con alegría: el valor de la virtud de la paciencia en tiempos de pandemia», propone el recurso a la narración y a sus héroes para contribuir a la formación del carácter. Tomando como referencia el pensamiento de santo Tomás, apela a la fortaleza, a la paciencia y a las virtudes en general como directrices básicas en la formación de la personalidad. Y las ejemplifica con Ulises, símbolo del amor a la patria y a la familia, quien resistió y venció todos los obstáculos hasta llegar a Ítaca. Su viaje de veinte años apela a la paciencia y a la perseverancia como cualidades que nos llevarán a alcanzar todas nuestras metas, a pesar de las dificultades del viaje.

En la misma línea Cintia Carreira, en «Los clásicos de la literatura ante la adversidad: Héctor y la fortaleza», ofrece un pormenorizado análisis literario de la Ilíada, que ayuda a 
comprender los distintos tipos de valentía presentes en ella, los motivos que mueven a sus personajes y la justificación antropológica de sus actos. Se atiende de manera particular al ejemplo de fortaleza de Héctor y también al de otros héroes, como Aquiles y Agamenón.

Cierra esta sección Marcin Kazmierczak con su ensayo «La lectura de grandes obras de la literatura universal como invitación al crecimiento personal en tiempos de calamidad. El proceso de la metanoia del personaje como paso hacia la resiliencia». El título asusta, pero se trata de una aportación lúcida y clara, a la que volveré a referirme infra.

La tercera sección consta de dos trabajos de lingüística. El primero es de Miguel Ángel Barbero Barrio, que en «Léxico del agradecimiento como factor de resiliencia para la vida post-COVID 19» considera la influencia en la conducta del léxico relacionado con la gratitud, que facilita un mejor actitud ante lo que llama «periodo post-COVID 19». Los hallazgos realizados en las últimas investigaciones -desde los campos lingüístico, neurobiológico y psicológico- apuntan de forma inequívoca hacia su propia interrelación.

Rafael Rodríguez-Ponga, en «El nacimiento de un nuevo vocabulario: consecuencias lingüísticas de la pandemia», aporta una excelente descripción del nuevo léxico covídico propio de esta catástrofe, del que son generadores principalmente los sanitarios y los medios de comunicación.

El cuarto bloque temático reúne cuatro aportaciones desde la pedagogía, que comparten un claro objetivo práctico: influir positivamente en todas las etapas educativas (desde la educación infantil hasta la universitaria), haciendo de la necesidad, virtud en estos tiempos calamitosos.

El primer capítulo, de Agustina Lacarte, se titula «Afrontar la educación a distancia de 0 a 17 años durante la época de confinamiento: cómo afecta a los niños y los adolescentes». Siguiendo la línea de esos cambios sociales y económicos experimentados, y del impacto que han supuesto para millones de niños la educación a distancia y el confinamiento, analiza cómo los centros educativos están cambiando su forma de enseñar para adaptarse a las nuevas circunstancias. Se describe también cómo el confinamiento afecta a los niños, y se analiza la resiliencia infantil como clave para superar y adaptarse a los cambios producidos.

Le sigue el trabajo de Raül Adames y Maria Turu, educadores, que lleva por título «Acción pedagógica y adaptación curricular desde los colegios CEU en Barcelona ante la emergencia de la pandemia». Los autores exponen en este capítulo la respuesta de los colegios CEU en Barcelona, siguiendo la estela de los retos a los que se enfrentan los centros educativos en la pandemia. Asimismo, se refieren a la acción pedagógica, que se ha centrado en una adaptación digital que permite mantener los pilares educativos, que son la relación profesor-alumno, la exigencia académica y la colaboración con las familias.

En el capítulo «Adaptaciones curriculares adoptadas por los centros educativos de la etapa de secundaria de la Comunidad de Madrid por COVID-19», María del Carmen Escribano Ródenas y Cándida Filgueira Arias, proponen cómo sacarle provecho a las tecnologías de la información y comunicación (TIC) de una manera muy diferente a como se venía haciendo hasta ahora. Las autoras enfatizan que es momento de redefinir estos nuevos parámetros con un aprendizaje humilde, y una reflexión sobre los cambios educativos y metodológicos que representan.

Por último, el capítulo de Sergio Rodríguez López-Ros, describe «La respuesta de la universidad a la situación de la pandemia: el caso de la Universitat Abat Oliba CEU». Ahí analiza la respuesta de la Universitat Abat Oliba CEU durante la crisis sanitaria a través de la 
dimensión docente, tutorial e investigadora. Así, se demuestra con evidencias que la actividad que conforma su modelo educativo se ha mantenido con total normalidad e incluso se ha intensificado a pesar de la distancia física, incrementando el apoyo al alumnado y el compromiso con la sociedad a la que presta servicio.

El quinto bloque lleva por título Métodos de la resiliencia ante la adversidad: aportaciones desde la Psicología. En «Resiliar en tiempos de crisis e incertidumbre», Laura Amado, Marina Fernández y Marta Oporto se aproximan a la resiliencia en la obra de los estudiosos más relevantes.

Estas mismas autoras, en el capítulo «Estrategias de afrontamiento y bienestar psicológico en la población infanto-juvenil en una situación de emergencia sanitaria» describen, como el título indica, la manera de afrontar en esta situación excepcional los peligros que acechan al equilibrio psicológico, emocional y relacional. Proponen estrategias concretas que conviene transmitir en el ámbito educativo.

Muy sugerente es la reflexión de Ermanno Pavesi en «Resiliencia: un enfoque personal». Tras exponer distintas visiones de la resiliencia desde la Antropología, y siguiendo las enseñanzas de Viktor Frankl, ofrece su propia reflexión, abierta a la trascendencia.

Los dos últimos estudios recogen consideraciones sobre la resiliencia desde el ámbito del derecho: Pablo Nuevo habla del «Coronavirus y derecho a la educación» y María Jesús Pesqueira de «Los derechos del alumno durante el estado de alarma. Especial atención a la privacidad en la realización de exámenes»; se trata, respectivamente, de una reflexión general y de un caso concreto de bien jurídico que debe ser protegido: la intimidad. Tratan un asunto que pasa inadvertido en la opinión pública. Estamos - también en los centros educativos de cualquier nivel- ante la clásica confrontación entre libertad y seguridad. Para prevenir los contagios no vale todo, sino que se deben salvaguardar derechos fundamentales; aquí, el derecho a la educación.

Sin preterir los méritos de los demás trabajos, quiero referirme más detenidamente al estudio de Marcin Kazmierczak, que parte del concepto de metanoia entendida como el proceso de cambio rápido y radical de la actitud del personaje hacia sí mismo y hacia los demás; esta le permite salir de su narcicismo y egocentrismo, y orientarse hacia una entrega de sí. A través de un cuidadoso estudio sobre distintos personajes de la literatura clásica se observa cómo, en la mayoría de casos, el motor de este cambio se encuentra en situaciones traumáticas y marcadas por la desgracia. Este proceso concluye en la catarsis como transformación resiliente, que puede transformar no solo al protagonista de estas historias, sino también a aquellos lectores que conozcan y se reconozcan en esas situaciones o en situaciones similares, tales como la situación de pandemia y las consecuencias que estamos viviendo.

Resumo: la primera de las aportaciones de este libro de carácter interdisciplinar es obvia: la propia interdisciplinariedad, tan predicada y tan poco practicada en el ámbito de las humanidades.

También constituye una crónica de acontecimientos de gran relevancia social (la mayor pandemia desde hace cien años), desde la perspectiva de la educación en sus varias dimensiones. Los autores son profesores de todos los niveles educativos, así como gestores de centros. Todos reflexionan, desde la primera fila de fuego en el combate, contra las consecuencias de la pandemia en el ámbito de la educación.

La mayoría de los textos, aunque partiendo de la plena conciencia de la gravedad de la situación, plantean diferentes vías de superación y medidas que pueden paliar, al menos 
parcialmente, las consecuencias de la pandemia y superarla exitosamente; en algunos casos, incluso descubriendo oportunidades en este escenario de por sí dramático. La mayoría de las propuestas giran en torno a alguno de los aspectos o los pilares de la resiliencia, entendida precisamente como una actitud de superación y de crecimiento en medio de circunstancias desfavorables.

Otro aspecto relacionado con la resiliencia -abordado sin complejos y deliciosamente incorrecto desde el punto de vista «político»- es el deseable crecimiento moral, tal y como podemos ver en el ejemplo de diversos personajes literarios o históricos que se vieron abocados a la desgracia individual o colectiva.

Estos trabajos fueron concebidos y elaborados hace unos meses, antes de la llegada de la segunda ola. Pienso que valdría la pena una segunda edición corregida y aumentada, que no hará sino avalorar la lucidez y la audacia que han demostrado los autores.

Francisco Crosas

Facultad de Humanidades de Toledo, Universidad de Castilla-La Mancha (UCLM). 